\title{
Characterizing Dynamic Transitions Associated with Snap-Through: A Discrete System
}

\author{
R. Wiebe \\ Graduate Research Assistant \\ Department of Civil Engineering \\ Duke University \\ Durham, NC 27708 \\ Email: rwiebe1313@gmail.com \\ I. Stanciulescu \\ Department of Civil Engineering \\ Rice University \\ Houston, TX 77251 \\ Email: ilinca@rice.edu
}

\author{
L.N. Virgin \\ Professor \\ Department of Mechanical Engineering \\ Duke University \\ Durham, NC 27708 \\ Email: I.virgin@duke.edu
}

S.M. Spottswood

Aerospace Engineer

Structural Mechanics Branch

AFRL/VASM, 2790 D Street WPAFB, OH 45433-7402

Email: Stephen.Spottswood@wpafb.af.mil

\author{
T.G. Eason \\ Aerospace Engineer \\ Structural Mechanics Branch \\ AFRL/VASM, 2790 D Street WPAFB, OH 45433-7402 \\ Email: Thomas.Eason@wpafb.af.mill
}

\begin{abstract}
Geometrically nonlinear structures often possess multiple equilibrium configurations. Under extreme conditions of excitation it is possible for these structures to exhibit oscillations about and between these co-existing configurations. This behavior may have serious implications for fatigue in the context of aircraft surface panels. Snap-through is a name often given to sudden changes in dynamic behavior associated with mechanical instability (buckling). This is an often encountered problem in hypersonic vehicles in which severe thermal loading and acoustic excitation conspire to create an especially hostile environment for structural elements. In this paper, a simple link model is used, experimentally and numerically, to investigate the mechanisms of snap-through buckling from a phenomenological standpoint.
\end{abstract}

\section{Introduction}

In order to meet ever increasing performance demands modern aircraft are being designed lighter than ever before. This weight efficiency usually leads to more slender structural components. Slender structures are susceptible to instabilities, particularly buckling, which can occur well before strength limits are reached. The nonlinear vibration of buckled structures may also lead to a large variety of chaotic and periodic oscillations. We are primarily interested in the circumstances under which a curved structure is forced to its inverted configuration, as shown in Fig. 1(a) and (b).

Snap-through buckling is a particular type of instability where the structure snaps from one state to another (remote) state. Such large deflections pose a hazard since they can exacerbate fatigue [1,2]. The two primary types of snap-through buckling, post buckled/bifurcated snap-through and limit point snap-through, are shown in parts (c) and (d) of Fig. 1 [3].

Limit point buckling occurs in structures where the stiffness decreases (with increasing loading) to a vanishing point, i.e., a horizontal tangency in the force-deflection relation (as shown in Fig. 1(c)). At the limit point the structure jumps to the remaining stable equilibrium. At loads below the limit point the structure may also snap-through under external perturbations. Postbuckled snap-through occurs in axially loaded structures, where a structure snaps between the two (often) symmetric buckled states under some perturbation, for example, from point $\delta_{e}$ to $\delta_{e}^{*}$ in Fig. 1(d). Postbuckled snap-through 


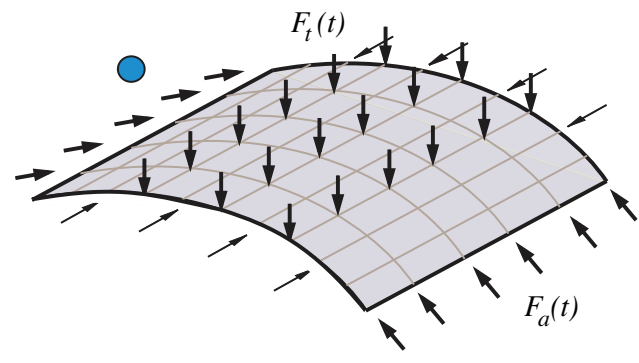

(a)

(b)
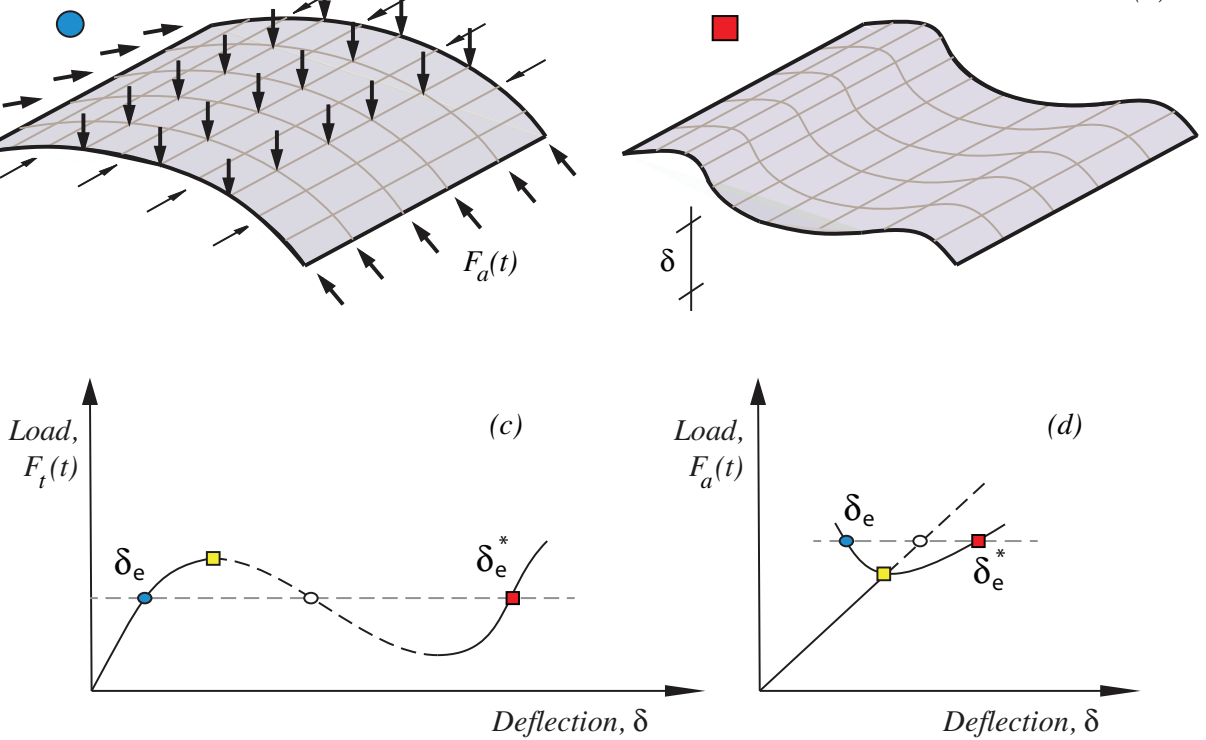

Fig. 1. A typical curved panel, and two schematic scenarios in which such a system might exhibit a snap-through event in its forcedisplacement relationship. (a) pre-snap, (b) post-snap, (c) limit point buckling, (d) pitchfork bifurcation.

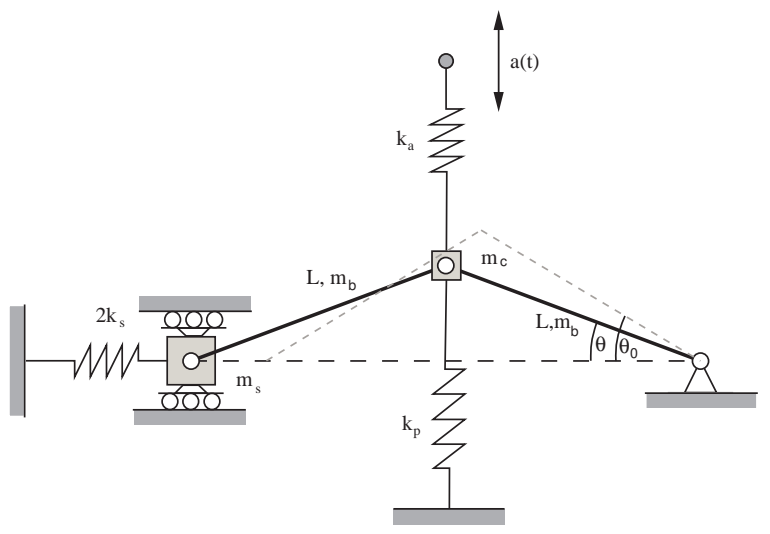

Fig. 2. A single degree of freedom (SDOF) link model.

typically occurs after pitchfork buckling; and it often requires that the structure first be loaded to a buckled configuration (as shown in Fig. 1(d)). Related behavior can also occur in aircraft surface panels that may buckle due to thermal loading, [4-6].

When a structure with an underlying snap-through static behavior is subjected to excitation, then highly nonlinear oscillations are possible. In order to gain phenomenological insight into this type of response and enable relatively unambiguous experimental verification we introduce a very simple single degree of freedom (SDOF) discrete structural system (Fig. 2). This system, for a certain induced pre-stress through the axial spring $2 k_{s}$, and $a(t)=$ constant (i.e., the actuator is held in a fixed position), possesses two symmetric static equilibria, $\theta= \pm \theta_{e}$. Furthermore, when the system is subject to 'lateral' excitation $a(t)$, we observe the possibility of complicated behavior involving motion that is heavily influenced by the locations of the underlying equilibria. A key aspect of this form of dynamic buckling is the existence of an unstable equilibrium between the stable states. These unstable equilibria define the basins of attraction for the stable states. Hence, the energies of the stable and unstable states may be useful for distinguishing between trajectories that snap from those that do not. The static equilibria, both stable and unstable, are therefore of great importance in studying the systems' forced dynamics. This system will be scrutinized using classical analysis techniques as well as experimental verification to shed light on the issue of dynamic snap-through in the larger context of aircraft structural components. Later work will focus on the multi-degree-of-freedom system, in which mode shapes, asymmetric behavior, etc. will be important features.

Single degree of freedom systems, particularly ones with Duffing-type potential wells, are well represented in the literature. An excellent review of the behavior of the forced Duffing oscillator is available in [7,8]. The route to potential well escape (i.e., snap-through for a mechanical system) is studied in [9] using Melnikov theory. Melnikov theory is also used in [10] to give a criterion for the existence of chaos in quasi-periodically forced Duffing oscillator. An SDOF structure with an underlying Duffing potential well was studied in [11]. This paper discusses the use of homoclinic orbits as the 

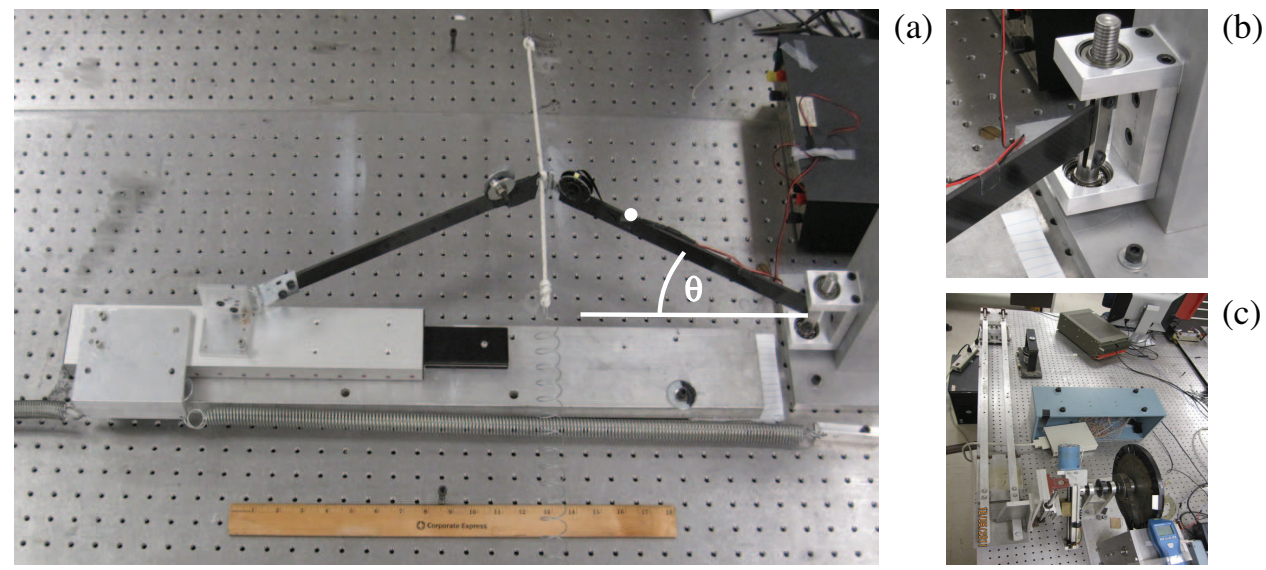

Fig. 3. (a) Photograph of experimental setup, (b) the low-friction pin joint, (c) the Scotch-yoke forcing mechanism.

bifurcation indicator in an unforced system, and bifurcation to and from chaos with respect to harmonic forcing parameters. A (modified) Duffing oscillator was studied numerically in $[12,13]$. In the vicinity of basin boundaries, small changes in initial conditions, parameters, or indeed in the numerical algorithm used, may lead to different attractors. A study of the bifurcations in forcing parameter space, similar to the goal of this research, is done in [14]. This work produced approximate analytical boundaries separating bounded from unbounded motion.

\section{Experimental Setup}

Shallow arches typically snap-through in a symmetric mode shape [15-19], and thus a SDOF link arch may be used to qualitatively model this behavior. For deeper arches, i.e., arches with a higher rise-to-span ratio, an asymmetric mode of buckling is typically encountered. Figure 3 is a photograph of the experimental SDOF link model tested. The two link arms were both carbon fiber and had a length of $32.4 \mathrm{~cm}$ and a mass of $41 \mathrm{~g}$. The center joint had a mass of $166 \mathrm{~g}$ and the slider had a mass of $1260 \mathrm{~g}$. Note that the system was set up horizontally on a test bed and hence force due to gravity was out of the plane of the system dynamics.

The two axial springs are an essential component of the structure and result in the underlying multivalued potential energy surface which mimics a shallow arch. As a practical consideration it was decided to use two opposing mutually pretensioned springs (each with $k_{s}=74 \mathrm{~N} / \mathrm{m}$ ) to avoid inducing compressive forces, which would of course buckle the springs. The two transverse springs connected to the center joint provided a transmitted force to the system via the displacement of the opposite end of the active spring, $k_{a}$. The displacement was provided by a Scotch-yoke mechanism moving with an approximately sinusoidal motion [8]. The second (anchor) spring, $k_{p}$, ensured a pre-tension in the active spring. The active and anchor springs had stiffnesses of $k_{a}=38 \mathrm{~N} / \mathrm{m}$ and $k_{p}=23 \mathrm{~N} / \mathrm{m}$ respectively. The control parameters for the system were the Scotch-yoke amplitude and frequency which equate to the forcing amplitude and forcing frequency of the system.

The most natural generalized coordinate for this system is the angle of the link arm on the non-sliding side of the structure. This angle, $\theta$, was measured using a high speed camera (Prosilica GC640). The arm position, and hence the angle, was obtained by locating a small white target on the link arm (see Fig. 3) by its contrast with the background using LabVIEW software. This process was done in real-time for each frame taken by the camera, providing a time series of the arm angle. Experimental results are discussed later in sections 4 and 5.

\section{Modeling}

A numerical model was developed to compare with the experimental results. Referring back to Fig. 2, we model the discrete dynamic system using an energy approach. Here, $m_{b}$ is the mass of the link arms, $m_{c}$ is the mass of the center joint (assumed to be a point mass), $m_{s}$ is the mass of the slider assembly and joint, $L$ is link length, $k_{s}$ are the lateral structural spring stiffnesses, $k_{a}$ is the active spring stiffness, $k_{p}$ is the anchor spring stiffness, and $\theta$ is the angle of the link arm on the non-sliding side. The spring masses were considered negligible. The displacement at the end of the active spring $a(t)$ is transmitted as a force through the active spring to provide the system forcing. The angle $\theta_{0}$ is the unforced equilibrium angle, i.e., the angle of the stable equilibrium with the forcing in the neutral position. Once again the effect of gravity is ignored in the analysis since the experimental system was laying flat. The kinetic and potential energies, along with the energy dissipation of the system are given by 


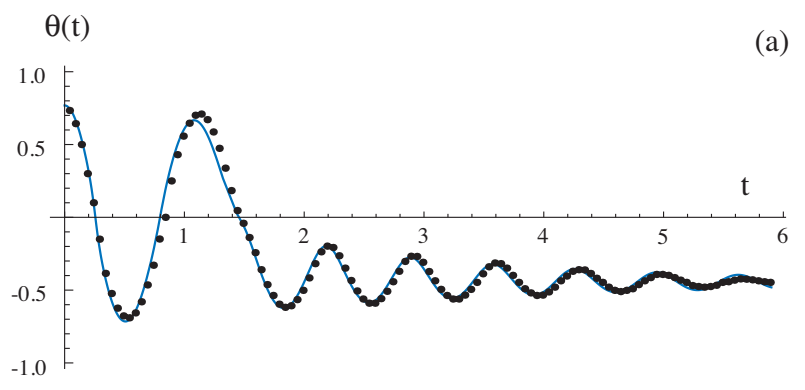

(b)

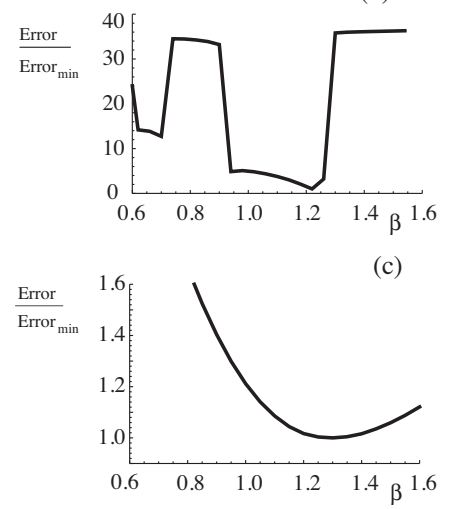

Fig. 4. Identification of damping parameter $\beta(\mathrm{Kg} / \mathrm{s})$. (a) A typical nonlinear free decay; the points are experimental data, and the continuous line represents the numerical integration of equation (4) with $\beta=1.2$. (b) Normalized average error vs. $\beta$ for the large amplitude time series (in part (a)). (c) Normalized average error vs. $\beta$ for a small amplitude time series.

$$
\begin{aligned}
T(\theta, \dot{\theta}, t)= & \left\{\frac{1}{2}\left(m_{c}+\frac{1}{3} m_{b}\right) L^{2}+\frac{1}{2} m_{s} x_{s}^{\prime}(\theta)^{2}\right. \\
& \left.+\frac{1}{6} m_{b}\left[x_{c}^{\prime}(\theta)^{2}+x_{c}^{\prime}(\theta) x_{s}^{\prime}(\theta)+x_{s}^{\prime}(\theta)^{2}+y_{s}^{\prime}(\theta)^{2}\right]\right\} \dot{\theta}^{2}, \\
V\left(\theta, t ; \theta_{1}\right)= & k_{s}\left[x_{s}(\theta)-x_{s}\left(\theta_{1}\right)\right]^{2}+\frac{1}{2} k_{a}\left[a(t)-y_{c}(\theta)\right]^{2}+\frac{1}{2} k_{p} y_{c}(\theta)^{2}, \\
F(\theta, \dot{\theta}, t)= & \frac{1}{2} \beta\left[\dot{x}_{s}(\theta)\right]^{2}=\frac{1}{2} \beta\left[x_{s}^{\prime}(\theta) \dot{\theta}\right]^{2},
\end{aligned}
$$

where $x_{c}(\theta)=L \cos (\theta), y_{c}(\theta)=L \sin (\theta), x_{s}(\theta)=2 L \cos (\theta)$. The masses of the springs were small and their contribution to the kinetic energy was neglected. The slider mechanism was by far the largest contributor to the damping of the experimental system; therefore the dissipation function $F$ was set to be a function of $\dot{x}_{s}$ only. The angle $\theta_{1}$ is the angle at which the two lateral structural springs were in equilibrium. This is the angle at which the potential energy of these two springs is at a minimum, i.e., the stable equilibrium of the system if the transverse (forcing) springs were removed. However the equilibrium states observed experimentally are at the stationary points of the total potential energy [20]. The two angles $\theta_{0}$ and $\theta_{1}$ are related by the expression

$$
\frac{\partial}{\partial \theta}\left[V\left(\theta ; \theta_{1}\right)\right]_{\theta=\theta_{0}}=0
$$

Note that the two forcing springs, $k_{a}$ and $k_{p}$, were set to be in equilibrium for $\theta=0$.

The potential and kinetic energy equations may be substituted into the Euler-Lagrange equation [21]

$$
\frac{d}{d t}\left(\frac{\partial(T-V)}{\partial \dot{\theta}}\right)-\frac{\partial T}{\partial \theta}+\frac{\partial V}{\partial \theta}+\frac{\partial F}{\partial \dot{\theta}}=0
$$

to yield the equation of motion of the system:

$$
\begin{aligned}
& {\left[m_{c}+\frac{2}{3} m_{b}+2\left(m_{b}+2 m_{s}\right) \sin ^{2} \theta\right] \ddot{\theta}+\left(m_{b}+2 m_{s}\right) \sin (2 \theta) \dot{\theta}^{2}+4 \beta \dot{\theta} \sin ^{2} \theta} \\
& \left.+8 k_{s}\left(\cos \theta_{1}-\cos \theta\right) \sin \theta+\frac{1}{2}\left(k_{a}+k_{p}\right) \sin 2 \theta-\left(k_{a} / L\right) \cos \theta\right) a(t)=0 .
\end{aligned}
$$

The damping coefficient $\beta(\mathrm{Kg} / \mathrm{s})$ was determined by fitting a simulated (using fourth-order Runge-Kutta time stepping of Eq. (4) with $\Delta t=0.001 \mathrm{~s}$ ) free decay response with the experimental response. In order to check the numerical stability of the Runge-Kutta routine it was also compared with simulations done using the NDSolve function available in Mathematica, with excellent agreement. Figure 4 (a) shows this comparison between simulated and experimental time series (for $\theta_{0}=26.0^{\circ}$ and $\theta_{1}=36.2^{\circ}$ ). We note that the agreement is good, despite the large-amplitude, snap-through characteristic of the free decay. Parts (b) and (c) of this figure show the sensitivity of the simulation error norm (calculated as the average of the 
absolute difference between simulated and experimental time series up to $t=6 \mathrm{~s}$ ) as a function of $\beta$. Part (b) is for the large amplitude experimental data points shown in part (a). The large jumps in the error occur because as the damping is changed the system will snap-through either too often (damping too low), or not enough (damping too high). The lower error region near $\beta=0.65$ occurs because the system in fact snaps-through an extra two times and therefore returns to the same side as the experimental data so the error is actually lower than for higher values of $\beta$ where only one extra snap event occurs. This leads to a smaller error norm even though it is less accurate. Note that although the system seems to be very sensitive to the damping coefficient, this plot corresponds to a single experimental time series. Finally part (c) shows a similar sensitivity study done using a small non-snapping free decay, which shows a clear minimum. Parts (a) and (b) hint that an optimal damping is approximately $\beta=1.2$, whereas part (c), for the small amplitude decaying oscillation, has a optimal value closer to $\beta=1.3$. This indicates that the system damping is slightly larger for small oscillations. However, since the results to be shown tended to be dominated by large amplitude oscillations the value $\beta=1.2$ was used throughout. The model could be refined by including the effects of Coulomb damping such as in [22], where Coulomb friction is shown to induce follower forces on the system. However, due to the overall excellent agreement between the experimental and numerical results shown later, it was decided that the viscous friction used in the model is accurately portraying the system dynamics in general, including energy dissipation.

\section{Equilibria and Stability}

The static force-displacement relationship may be obtained by finding the static force (or in this case static displacement $\left.a(t) \rightarrow a_{e}\right)$ and angle $\theta_{e}$ for which the potential energy of the system exhibits a stationary point. The force-displacement relationship is given by

$$
a_{e}=\frac{L}{k_{a}}\left[k_{a}+k_{p}+8 k_{s}\left(\frac{\cos \theta_{1}}{\cos \theta_{e}}-1\right)\right] \sin \theta_{e} .
$$

According to the theorem of minimum potential energy the stability of the equilibria are given by the sign of the second derivative of the potential energy function [20]. A positive (negative) second derivative implies a minimum (maximum) potential and hence stable (unstable) equilibrium. An equivalent approach is to investigate the local natural frequency of the system for small oscillations about the equilibria under the static load being considered $[3,23]$. This method is preferred because unlike potential energy it is easy to directly measure the response frequency of an experimental system. The natural frequency may also be of interest in its own right. The sign of $\omega^{2}$ determines stability, i.e., if $\omega^{2}>0$ then disturbances do not grow in time. Linearizing the equation of motion about a static equilibrium position $\theta=\theta_{e}$ under a static load $a(t)=a_{e}$ produces the equation of a simple harmonic oscillator from which the undamped natural frequency is easily determined to be

$$
\omega_{0}^{2}=\frac{8 k_{s} L^{2} \cos \theta_{1} \cos \theta_{e}+\left(k_{a}+k_{p}-8 k_{s}\right) L^{2} \cos 2 \theta_{e}+k_{a} L a_{e} \sin \theta_{e}}{\frac{1}{2}\left(m_{c}+\frac{1}{3} m_{b}\right) L^{2}+\frac{1}{3} m_{b} L^{2}+2\left(m_{b}+2 m_{s}\right) L^{2} \sin ^{2} \theta_{e}},
$$

where the numerator is the second derivative of the potential energy. Given that the denominator of equation (6), i.e., the inertia, is positive definite, this result is clearly in agreement with the theorem of minimum potential energy.

Figure 5 shows the relationship between the force (static displacement of the actuator $a_{e}$ ), displacement, and local natural frequency for both the experimental system and the analytical model (for $\theta_{0}=26.0^{\circ}$ and $\theta_{1}=36.2^{\circ}$ ). The experimental response frequency is necessarily the damped natural frequency, whereas in the analytical result is the undamped natural frequency. However, because the damping is relatively small these two values are still very close. For the experimental results there are no data points in the unstable region given that these equilibria cannot be observed. However the agreement is excellent in the stable region.

The static equilibria of dynamical systems, and underlying potential energy, give much insight into the dynamic response, i.e., they act as an organizing framework for the nonlinear behavior. If the system is subject to periodic excitation, they also influence large amplitude snap-through oscillations and highly nonlinear global behavior. This will be considered in the next section.

\section{Dynamic Response}

The forced dynamic response of the system was simulated by applying a fourth-order Runge-Kutta time stepping scheme on Eq. (4) with $a(t)=A \sin \Omega t$. Figure 6 shows some typical periodic responses from the system in Fig. 5 (numerical simulation in parts (a) and (b) and experimental in parts (c) and (d)). Each time series was produced with a forcing amplitude 


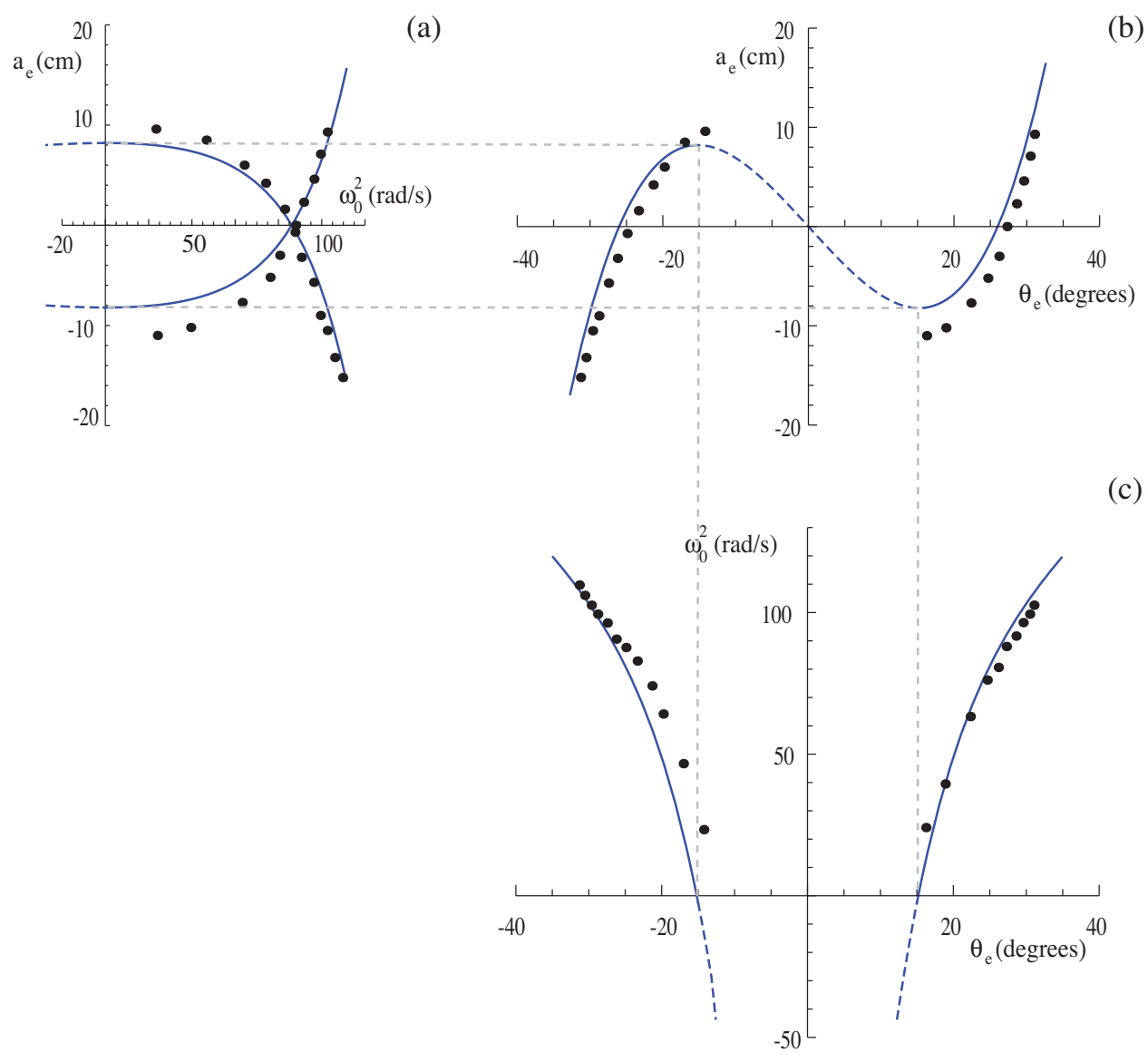

Fig. 5. Free response characteristics. (a) force vs. natural frequency (squared), (b) force vs. deflection, (c) natural frequency (squared) vs. deflection. The points are experimental data, the continuous lines are the theoretical results.
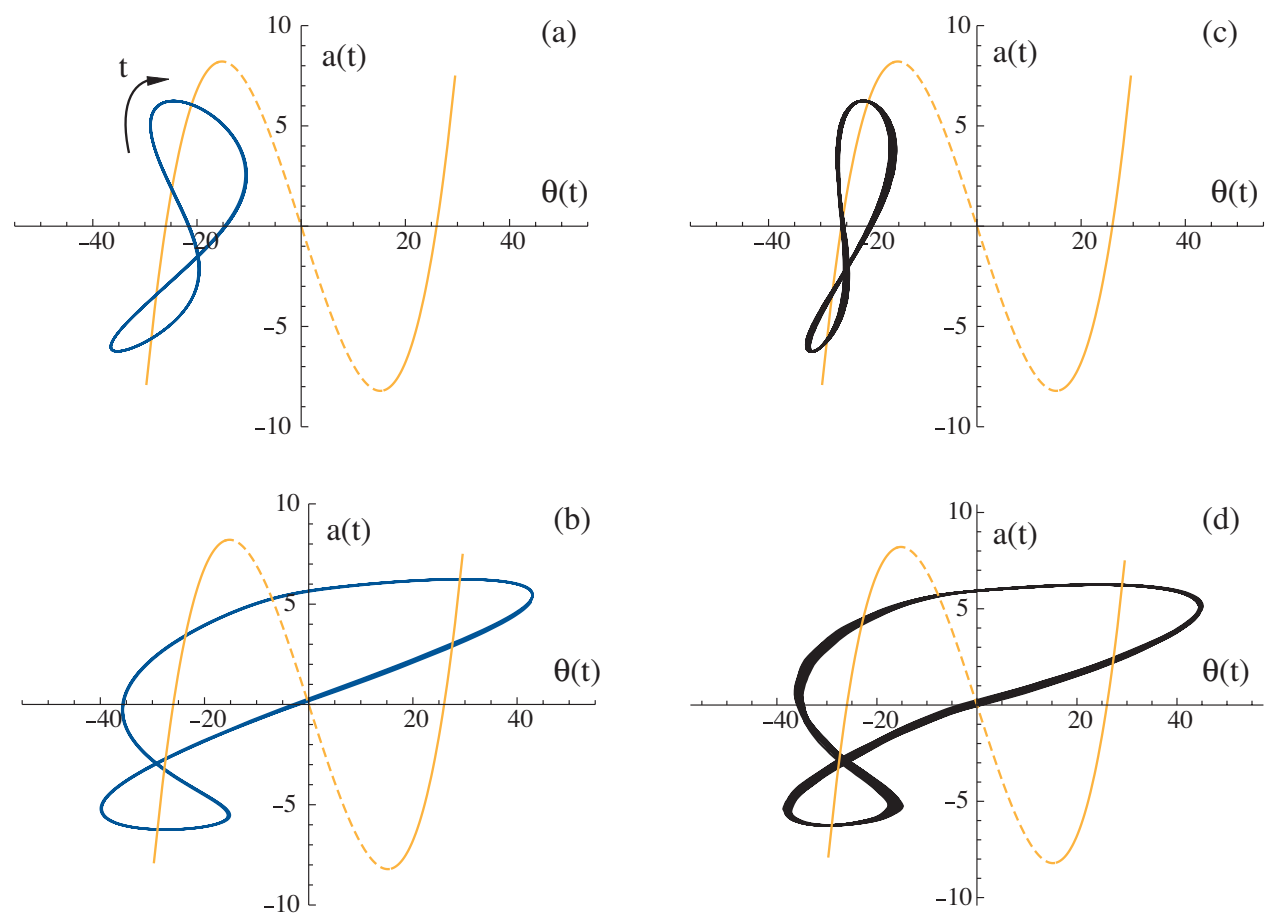

Fig. 6. Experimental and simulated time series superimposed on the restoring force. Numerical, (a) $\Omega=4.40 \mathrm{rad} / \mathrm{s}$; (b) $\Omega=3.36 \mathrm{rad} / \mathrm{s}$; Experimental, (c) $\Omega=4.40 \mathrm{rad} / \mathrm{s}$; (d) $\Omega=3.36 \mathrm{rad} / \mathrm{s}$. 

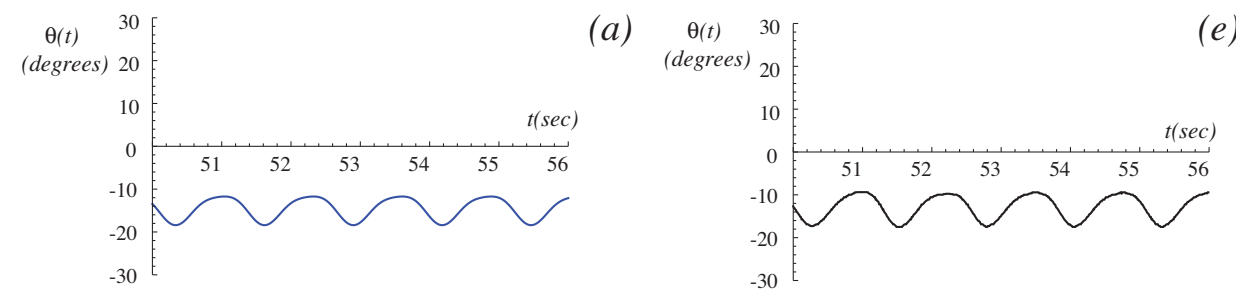

(e)

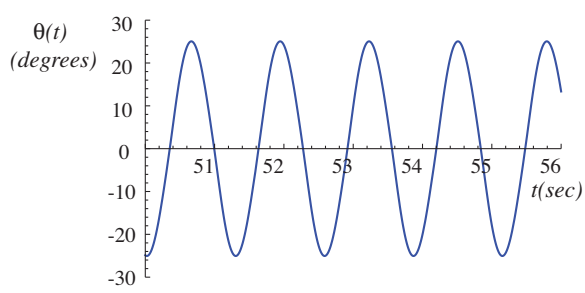

(b)
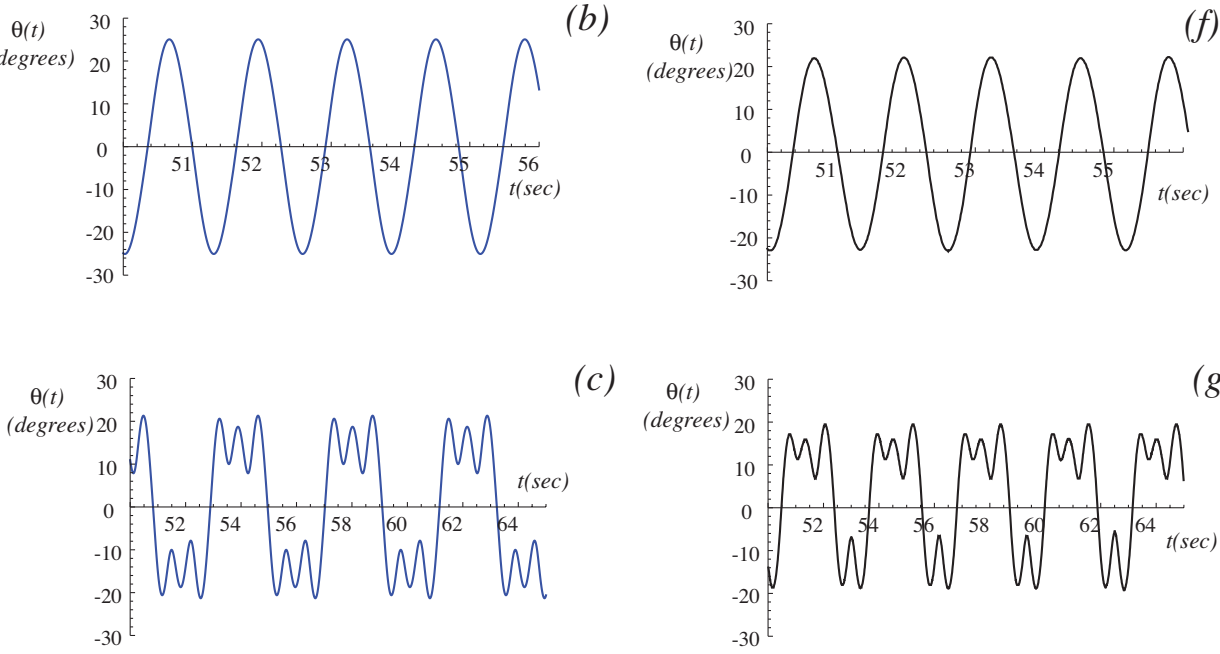

(c)
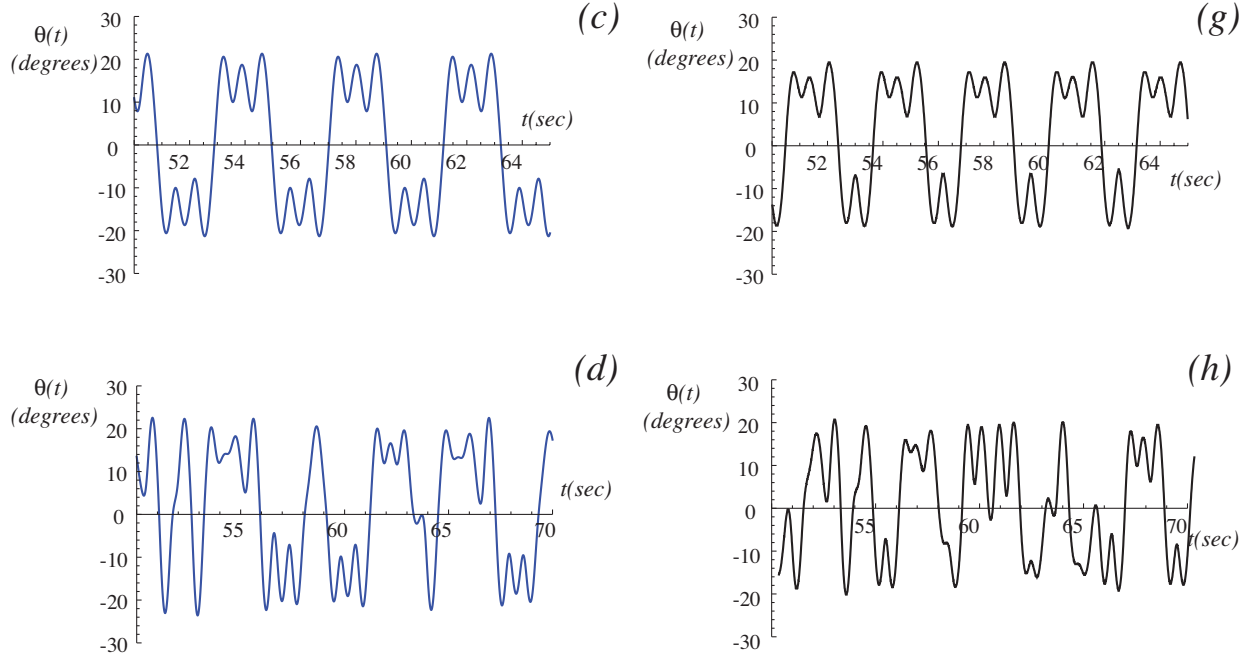

Fig. 7. Numerical (a-d) and experimental (e-h) time series. (a) $\Omega=4.9 \mathrm{rad} / \mathrm{s}$; (b) $\Omega=4.9 \mathrm{rad} / \mathrm{s}$; (c) $\Omega=7.6 \mathrm{rad} / \mathrm{s}$; (d) $\Omega=7.8 \mathrm{rad} / \mathrm{s}$; (e) $\Omega=4.9 \mathrm{rad} / \mathrm{s}$; (f) $\Omega=4.9 \mathrm{rad} / \mathrm{s}$; (g) $\Omega=7.9 \mathrm{rad} / \mathrm{s}$; (h) $\Omega=8.1 \mathrm{rad} / \mathrm{s}$.

of $A=6.25 \mathrm{~cm}$ (compare with the $A_{\text {snap }} \approx 8.21 \mathrm{~cm}$ ), however parts (a) and (c) corresponded to a forcing frequency of $\Omega=4.40 \mathrm{rad} / \mathrm{s}$ while parts (b) and (d) corresponded to $\Omega=3.36 \mathrm{rad} / \mathrm{s}$. The transmissibility of the forcing mechanism effectively translates to a moving restoring force, but superimposing the phase trajectories in this way gives a clear indication of whether the system is oscillating about, or snapping between, the two sides of the system. It is interesting that there is better agreement between the numerical and experimental results for the large snap-through motion in Fig. 6(b) and (d) than for the small non snap-through motion in (a) and (c). It is likely that this is due to some nonlinear damping effects that were not included in the model, particularly Coulomb damping (already discussed earlier), which has a more pronounced effect on slower motions.

Figure 7 shows several time series from simulation (a-d) and experiment (e-h), this time for a more shallow angle $\theta_{0}=15.0^{\circ}\left(\theta_{1}=29.9^{\circ}\right)$ and $A=1.10 \mathrm{~cm}$ (compare with $A_{\text {snap }} \approx 1.56 \mathrm{~cm}$ ). The relatively simple 'period-one (P1)', periodic responses shown in parts (a,b,e,f) correspond to a forcing frequency of $\Omega=4.9 \mathrm{rad} / \mathrm{s}$, i.e., they co-exist at this set of parameter values, and which attractor occurs depends on the initial conditions. This aspect of the behavior will be revisited a little later.

The non-simple but still periodic responses shown in parts ( $\mathrm{c}$ and $\mathrm{g}$ ) are not quite the same. The numerical result shown in part (c) occurred for $\Omega=7.6 \mathrm{rad} / \mathrm{s}$ and can be characterized as a 'period-four (P4)' oscillation, i.e., it repeats itself every four forcing cycles. The experimental result shown in part $(\mathrm{g})$ is for a slightly different forcing frequency $(\Omega=7.9 \mathrm{rad} / \mathrm{s})$ and is in fact a P5 oscillation. The chaotic behavior for the numerical result (part d) corresponds to $\Omega=7.8 \mathrm{rad} / \mathrm{s}$ and the experimental result (part h) corresponds to $\Omega=8.1 \mathrm{rad} / \mathrm{s}$. These values are very close to those used to generate the time series in parts (c and $\mathrm{g}$ ), thus suggesting a parameter sensitivity in this range.

The frequency content of these time series (see Fig. 8 for the more interesting time series) can be quite useful in assessing certain qualitative features of the dynamics. They can also assist in determining whether the system is exhibiting snap-through oscillations, since it is typically somewhat difficult to consistently distinguish between trajectories that snap- 

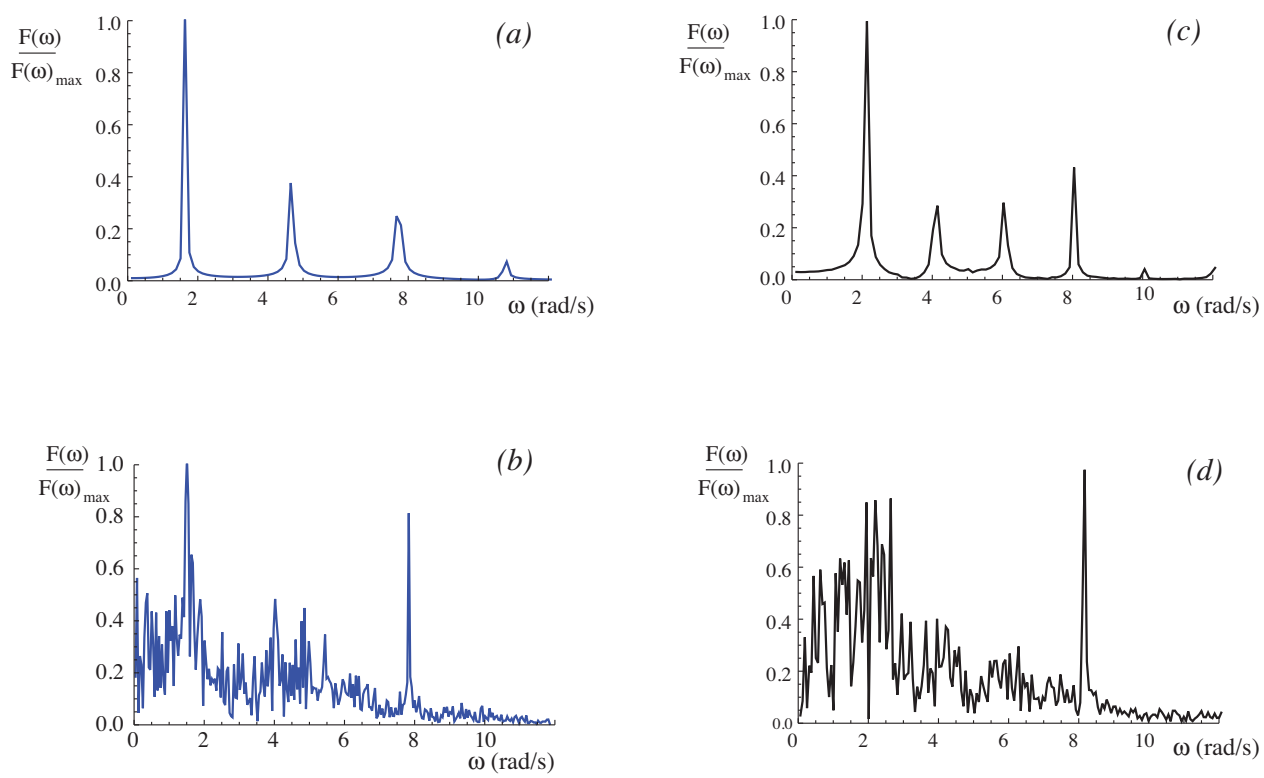

Fig. 8. Experimental and simulated DFT's for parts (c,d,g,h) in Fig. 7 respectively.
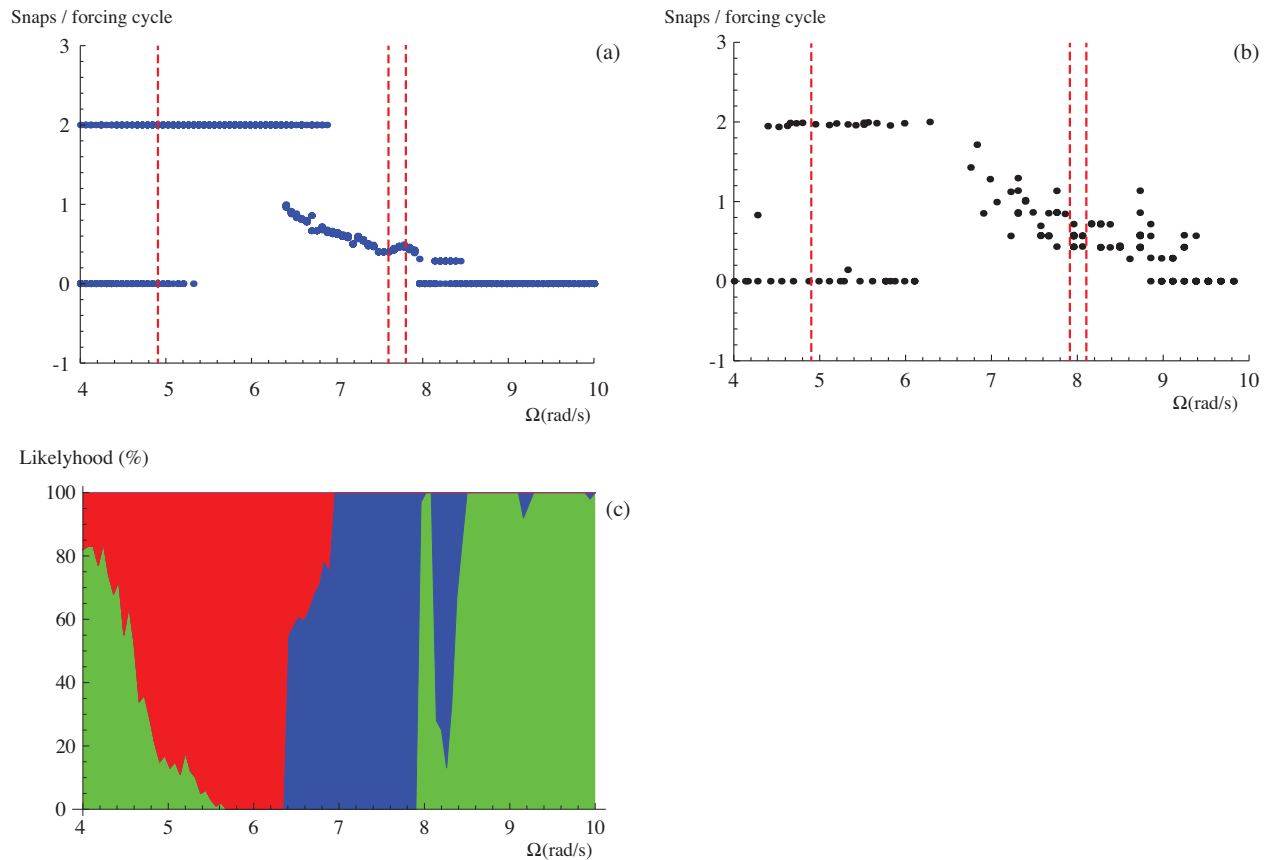

Fig. 9. Occurrence of snap-through. (a) simulation, (b) experiment, (c) relative dominance of co-existing attractors (simulation only). Green - non-snap, red - P1 snap-through, blue - higher periodic or chaotic (less frequent) snap-through. The vertical dashed red lines in (a) and (b) indicate the specific frequencies relating to Fig. 7 .

through from those that do not. The broadband nature of the discrete Fourier transform (DFT) of a time series is of course a well-established signature of chaos.

Figure 9 contains a 'bifurcation' diagram of snap-through behavior as a function of forcing frequency with $\left(\theta_{0}=15.0^{\circ}\right.$, $A=1.10 \mathrm{~cm}$ ). Parts (a) and (b) show the number of snap-through events per forcing cycle from simulation and experimental results, respectively. A snap-through event was considered to be any crossing of the state $\theta=0$. This definition of snapthrough works relatively well for the system because the unstable equilibria does not change significantly with the forcing. Each data point in part (a) indicates the result of one simulation with the snap-through events per forcing cycle being averaged over 1000 forcing cycles after the transients were given sufficient time to decay. The plot was created by running 10 simulations with random initial conditions for 100 forcing frequencies to capture co-existing attractors. The experimental data points were instead obtained by a performing a moving average of the number snap-through events per forcing cycle on a sweep-up followed by a sweep-down through forcing frequency. The sweep-up and -down primarily differ in the region 

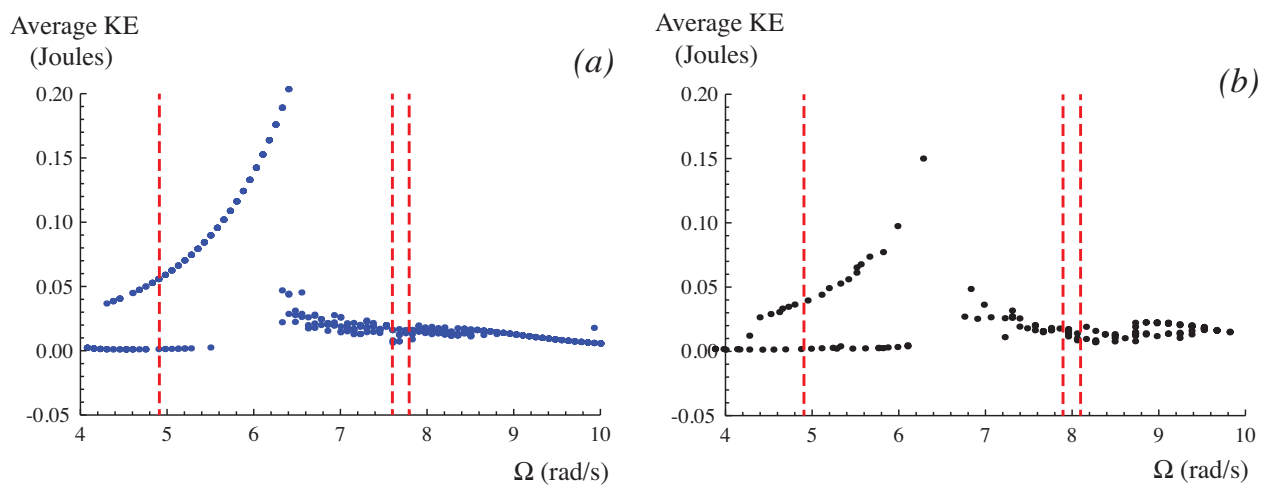

Fig. 10. Average kinetic energy as a function of forcing frequency, (a) simulation, (b) experiment.

below $\Omega=6.2 \mathrm{rad} / \mathrm{s}$ where the sweep-up did not snap-through and the sweep-down did. The responses that snap-through twice per forcing cycle indicate a snap-through in-phase with the forcing, i.e., P1 snap-through (see Fig. 7(b and f) for example time series). The responses with intermediate occurrences of snap-through indicate responses with occasional, or sporadic, snap-through, which may occur in higher-period periodic behavior (Fig. 7(c and g)) and chaotic behavior (Fig. 7(d and h)). Figure 9(c) shows the likelihood (via simulation only) of the system response converging to one of the three competing types of behavior seen it parts (a) and (b), where green corresponds to no snap-through, red corresponds to P1 snap-through, and blue is higher-periodic or chaotic (less frequent) snap-through. The likelihood was measured by running 100 simulations at random initial conditions at each frequency. This method may be more difficult to apply to higher dimensional systems as the separatrices are typically more complex and may be more dependent on the system forcing. However, in this relatively simple system it gives a straightforward interpretation of a potentially onerous event (snap-through) and thus useful data to inform practical issues including life-time fatigue predictions.

Another approach might be to observe some kind of energy measure of a given response. For a system to snap-through it is postulated that the external forcing must add a sufficient amount of energy to the system. Figure 10 shows the average kinetic energy over a span of forcing cycles against the forcing frequency for the same parameters as Fig. 9. The experimental data points were obtained by first sweeping slowly up, then down, through forcing frequency. The agreement between the experimental and numerical results is excellent, with only a slight horizontal shift in the data points.

One anticipates that the average kinetic energy of a system would be much larger for trajectories that traverse across the unstable equilibria than for those that oscillate about a single equilibria; however this detection is made more difficult by responses that only occasionally snap-through. This typically occurs with very high periodic, or more often, chaotic responses, which prevail for much of the right half of the energy plots. This makes the determination of chaos another important aspect of the analysis, especially since it is known that single well chaos is relatively rare [24].

The standard method of identifying chaos is to determine the Lyapunov spectrum of the system. Lyapunov exponents (LE) are however relatively difficult to determine from experimental data [25]. Figure 11(a) shows the LE obtained from the simulation of equation (4) (using the method in [26]) against forcing frequency. The data points were produced by running 10 simulations for different random initial conditions at 100 frequencies to make it possible to capture co-existing attractors. When comparing with Fig. 10 it appears that most of the chaotic responses of the system occur for snap-through trajectories.

An alternative approach is introduced in [27], whereby the number of distinct frequency spikes (peaks on the DFT, above a certain threshold) of a dynamical system are plotted against the parameter used for the simulation/experiment. There is typically a clear dichotomy in the number of peaks for a chaotic response with a broadband spectrum and a non-chaotic response with a finite number of peaks, making identification of chaos possible. Figure 11(b) shows the identification of chaos using this peak count method. In these results 10 simulations with randomly generated initial conditions were run at 100 forcing frequencies. It is confirmed that the chaotic responses are almost entirely within the region of high energy response.

An interesting 'second-order' feature in these plots is an assessment of how chaotic a signal might be. Two sample time series (indicated by the vertical dashed red lines) are also shown in Fig. 11(c) and (d). Part (d) corresponding to $\Omega=7.6$ $\mathrm{rad} / \mathrm{s}$ shows a slightly stronger periodicity than the time series shown in part (c) $(\Omega=7.0 \mathrm{rad} / \mathrm{s})$ : the second half of the time series appears nearly periodic, this short term periodicity is ubiquitous throughout the time series as the trajectory appears to spend more time oscillating about (rather than between) the underlying static equilibria. When subject to the LE algorithm this periodic signature results in a slightly less positive LE, and there are a few less spectral peaks occurring above the threshold. It is not uncommon for a chaotic trajectory to spend periods of time in a near-periodic state (a typical chaotic attractor has embedded within it many unstable periodic orbits). It was also just mentioned that in certain (relatively small) regimes of forcing frequency co-existing oscillations occur. In order to illuminate this feature, Fig. 11(e) shows the outcome of 100 simulations using randomly generated initial conditions, in which the percentage of numerical simulations 

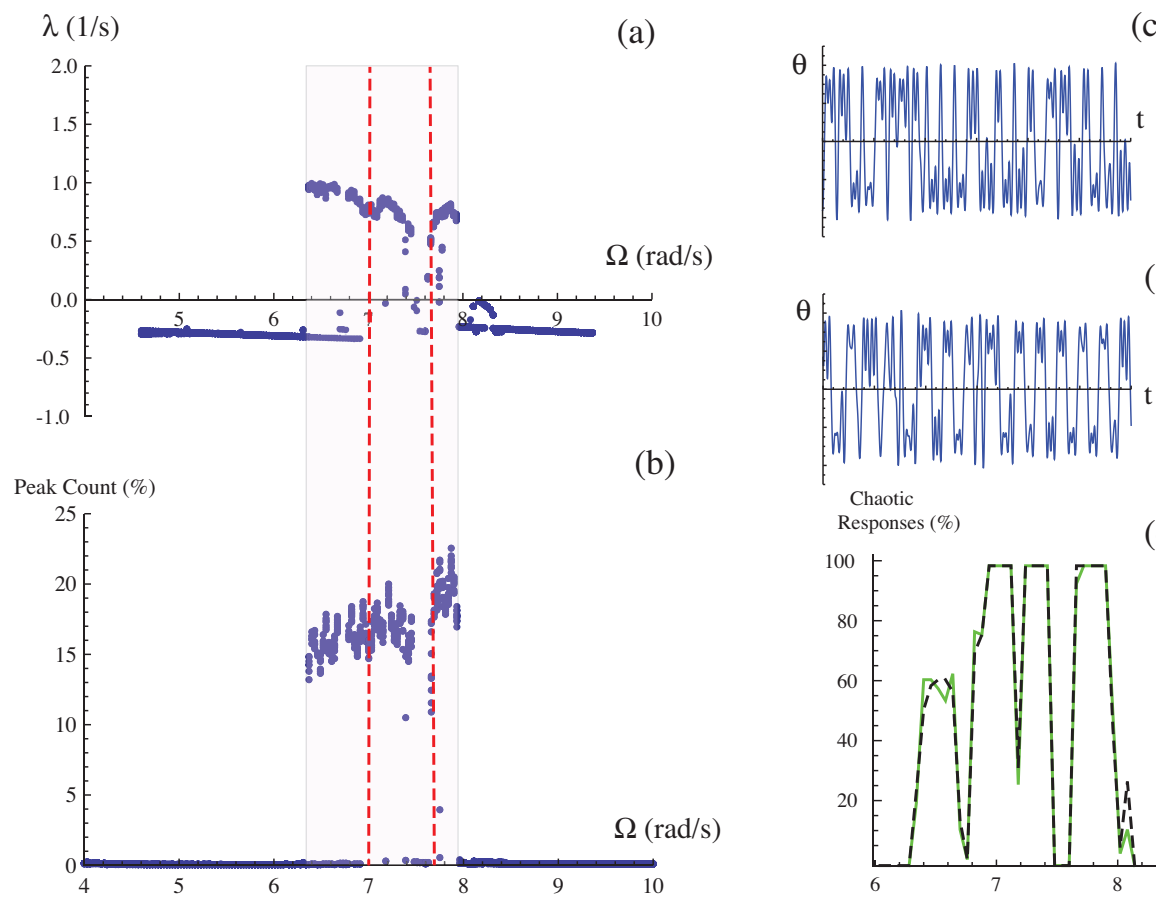

(c)

(d)
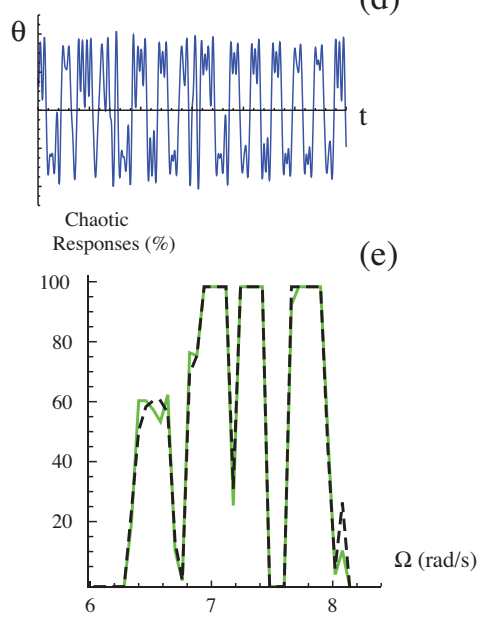

Fig. 11. Distinction between chaotic and non-chaotic behavior based on (a) the largest Lyapunov exponent, (b) the peak-count criterion; (c) and (d) typical chaotic time series, (e) relative dominance of chaotic behavior.

that lead to chaos is charted for both the LE (dashed black line) and the peak-count (solid green line) approaches. Clearly, both criteria are effective in distinguishing chaotic from non-chaotic motion. Outside of this range of forcing frequencies the behavior was typically non-chaotic, although co-existing attractors could still exist, and the periodic motion could be either small-amplitude (contained within the vicinity of an equilibrium), or relatively large-amplitude (cross-well) motion.

The same approach was also applied to the experimental system. Figure 12 shows bifurcation diagrams, based on 18 tests, in terms of chaotic vs. non-chaotic behavior. The computation of the largest Lyapunov exponent $(\lambda)$ is based on a standard algorithm [28] in which a time series is assessed in terms of the exponential rate at which nearest neighbors tend to evolve in time. This can be a sensitive undertaking, for example, part (a), for $\Omega=6.518$, and (b), for $\Omega=8.140$ of Fig. 12 show how the approach requires a linear fit. This is by no means straightforward. The red dashed lines in (a) and (b) show an approximately exponential divergence (the y-axis is a log-scale) for short time evolutions. However, the response in part (a) is characterized by a very small slope and corresponds to a periodic oscillation, whereas the case in part (b) is more convincingly positive and corresponds to a chaotic oscillation. Part (c) of this figure summarizes this behavior, and part (d) shows the equivalent results based on the peak count criterion. The red dashed lines in these parts show the specific frequencies at which the snapshots were taken for parts (a) and (b). The peak counts approach appears to be more robust and easier to apply than the conventional LE approach. The agreement with the numerical results is quite good. The bifurcations are slightly shifted, however the progression and even some of the periodic windows are still captured.

\section{Conclusions}

Snap-through buckling is investigated on a SDOF system. Some aspects of the underlying force-deflection and free vibration behavior of the system are described. Under the action of periodic excitation, the focus of interest shifts from equilibria to oscillatory behavior. In a broad sense, the behavior of the forced system can be divided into regimes of either small-amplitude or large-amplitude behavior. This latter response can be associated with snap-through behavior - an often highly undesirable behavior in many structural applications. Alternatively, the behavior can be divided into regimes of chaotic and non-chaotic behavior. Chaos is commonly associated with snap-through behavior but they are not necessarily the same thing. A promising method of distinguishing between small- and large-amplitude responses based on average total energy is introduced and applied to the system with good agreement between experimental results and the numerical model. Another method based on distinguishing between chaotic and non-chaotic responses is also proposed. In general, this work provide a useful overview of how snap-through behavior depends on the forcing parameters. 


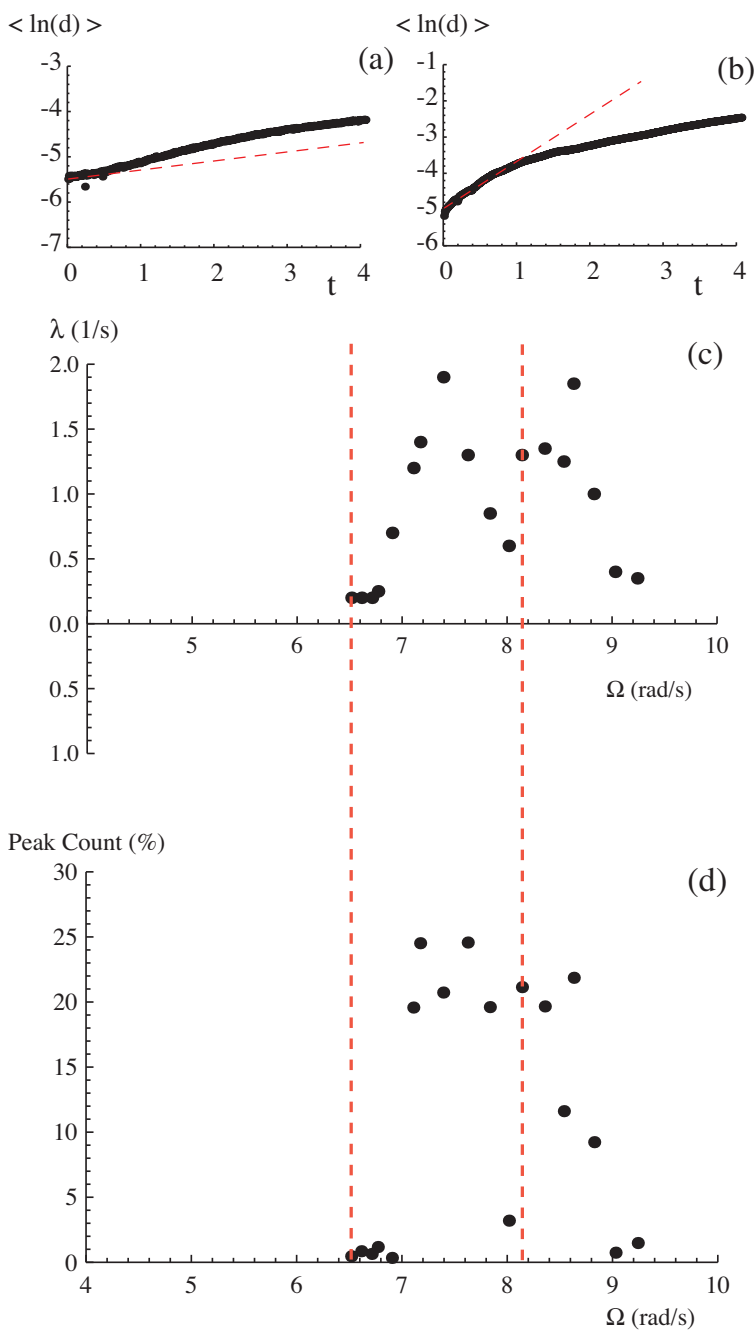

Fig. 12. Experimental LE and peak count. (a) and (b) typical linear fits for the local rate of divergence, (c) Largest LE as a function of the forcing frequency, (d) corresponding peak count result.

\section{Acknowledgments}

The authors acknowledge the support of Air Force Office of Scientific Research (AFOSR) grant FA9550-09-1-0204.

\section{References}

[1] B. Clarkson. Review of sonic fatigue technology. Tech. Report, NASA Contract Report 4587, 1994.

[2] S. Suresh. Fatigue of Materials, 2nd edition. Cambridge University Press, 1998.

[3] L. N. Virgin. Vibration of Axially Loaded Structures. Cambridge University Press, 2007.

[4] S. P. Timoshenko and S. Woinowsky-Krieger. Theory of Plates and Shells, 2nd Edition. McGraw Hill, 1959.

[5] L. W. Chen and L. Y. Chen. Thermal buckling of laminated composite plates. Journal of Thermal Stresses, 10(4):345$356,1987$.

[6] K. D. Murphy, L. N. Virgin, and S. A. Rizzi. The effect of thermal prestress on the free vibration characteristics of clamped rectangular plates: theory and experiment. Journal of Vibration and Acoustics, 119:243-249, 1997.

[7] Y. Ueda. Survey of regular and chaotic phenomena in the forced Duffing oscillator. Chaos, Solitons \& Fractals, 1:199, 1991.

[8] L. N. Virgin. Introduction to Experimental Nonlinear Dynamics. Cambridge University Press, 2000.

[9] J. M. T. Thompson. Fractal basins and chaotic bifurcations prior to escape from a potential well. J. M. T. Thompson and S. R. Bishop and L. M. Leung, 121:116, 1987.

[10] S. Wiggins. Chaos in the quasiperiodically forced Duffing oscillator. Physics Letters A, 124:138, 1987.

[11] I. Ario. Homoclinic bifurcation and chaos attractor in elastic two-bar truss. International Journal of Nonlinear Mechanics, 39:605, 2004. 
[12] P. S. Addison, A. H. C. Chan, D. A. Ervine, and K. J. Williams. Observations on numerical method dependent solutions of a modified Duffing oscillator. Communications in Applied Numerical Methods, 8:519, 1992.

[13] L. Bardella and F. Genna. Newmark's time integration method from discretization of extended functionals. Journal of Applied Mechanics, 72:527, 2005.

[14] A. H. Nayfeh and N. E. Sanchez. Bifurcations in a forced softening Duffing oscillator. International Journal of Nonlinear Mechanics, 24:483, 1989.

[15] C. Hsu. Stability of shallow arches against snap-through under timewise step loads. Journal of Applied Mechanics, 35:31-39, 1968.

[16] L. Rehfield. Nonlinear flexural oscillations of shallow arches. AIAA Journal, 12:91-93, 1974.

[17] E. Johnson and I. McIvor. The effect of spatial distribution on dynamic snap-through. Journal of Applied Mechanics, 45:612-618, 1978.

[18] W. Gregory and R. H. Plaut. Dynamic stability boundaries for shallow arches. Journal of Engineering Mechanics, 108:1036-1050, 1982.

[19] R. H. Plaut and J. C. Hsieh. Oscillations and instability of a shallow arch under two-frequency excitation. Journal of Sound and Vibration, 102:189-201, 1985.

[20] J. M. T. Thompon and G. W. Hunt. A General Theory of Elastic Stability. Wiley, 1973.

[21] H. Goldstein, C. P. Poole, and J. L Safko. Classical Mechanics (3rd Ed.). Addison Wesley, 2001.

[22] D. Bigoni and G. Noselli. Experimental evidence of flutter and divergence instabilities induced by dry friction. Journal of the Mechanics and Physics of Solids, To appear.

[23] L. N. Virgin. Parametric studies of the dynamic evolution through a fold. Journal of Sound and Vibration, 110:99-109, 1986.

[24] J. A. Gottwald, L. N. Virgin, and E. H. Dowell. Experimental mimicry of Duffing's equation. Journal of Sound and Vibration, 158:447-467, 1992.

[25] G. Q. Wu, N. M. Arzeno, L. L. Shen, D. K. Tang, D. A. Zheng, N. Q. Zhao, D. L Eckberg, and C. S. Poon. Chaotic signatures of heart rate variability and its power spectrum in health, aging and hear failure. Plos One Open Access, $4: 1-9,2009$.

[26] W. C. Xie. Dynamic Stability of Structures. Cambridge University Press, 2006.

[27] R. Wiebe and L. N. Virgin. A heuristic method for identifying chaos from frequency content. Chaos, To appear.

[28] M. T. Rosenstein, J. J. Collins, and C. J. D. Luca. A practical method for calculating the largest Lyapunov exponents from small data sets. Physica D: Nonlinear Phenomena, 65:117-134, 1993. 